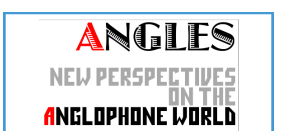

ANELOPHONE WORLI

\section{Angles}

New Perspectives on the Anglophone World

$12 \mid 2021$

COVID-19 and the Plague Year

\title{
Diamond Princess II
}

A Graphic Tale

\section{Amine Barbuda}

\section{(2) OpenEdition}

Journals

Electronic version

URL: https://journals.openedition.org/angles/3580

DOI: $10.4000 /$ angles.3580

ISSN: 2274-2042

\section{Publisher}

Société des Anglicistes de l'Enseignement Supérieur

\section{Electronic reference}

Amine Barbuda, "Diamond Princess II", Angles [Online], 12 | 2021, Online since 01 May 2021, connection on 22 December 2021. URL: http://journals.openedition.org/angles/3580 ; DOI: https://doi.org/

10.4000 /angles. 3580

This text was automatically generated on 22 December 2021.

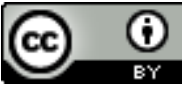

Angles est mise à disposition selon les termes de la Licence Creative Commons Attribution 4.0 International. 


\section{Diamond Princess II}

A Graphic Tale

Amine Barbuda

Figure 1: Prologue, Diamond Princess II

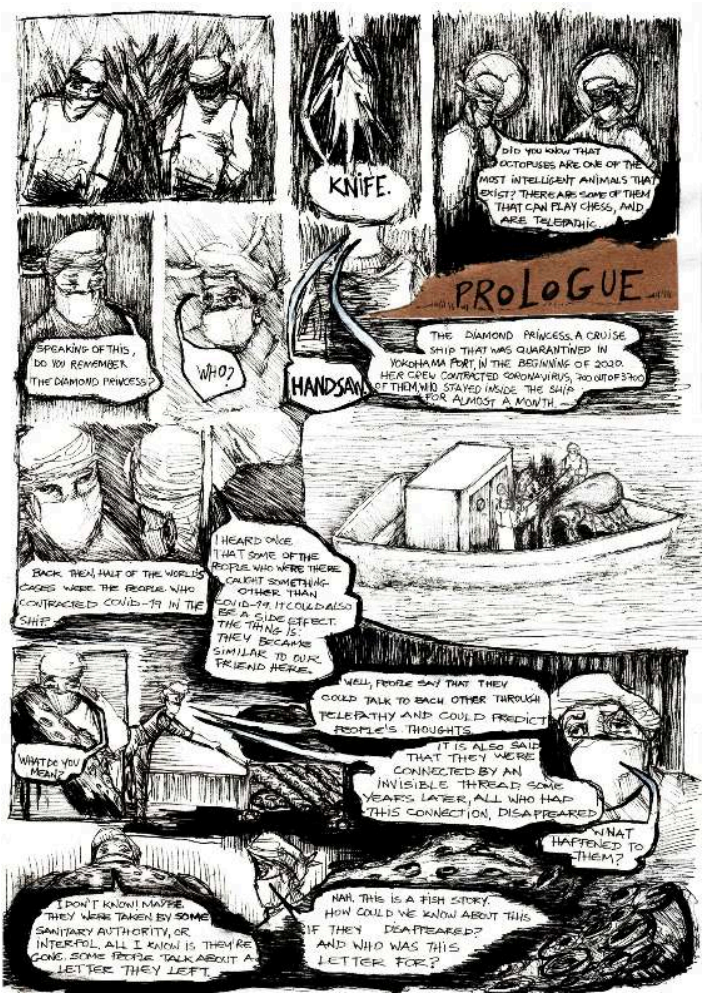

Credits: Amine Barbuda. 
Figure 2: Plate 1, Diamond Princess II

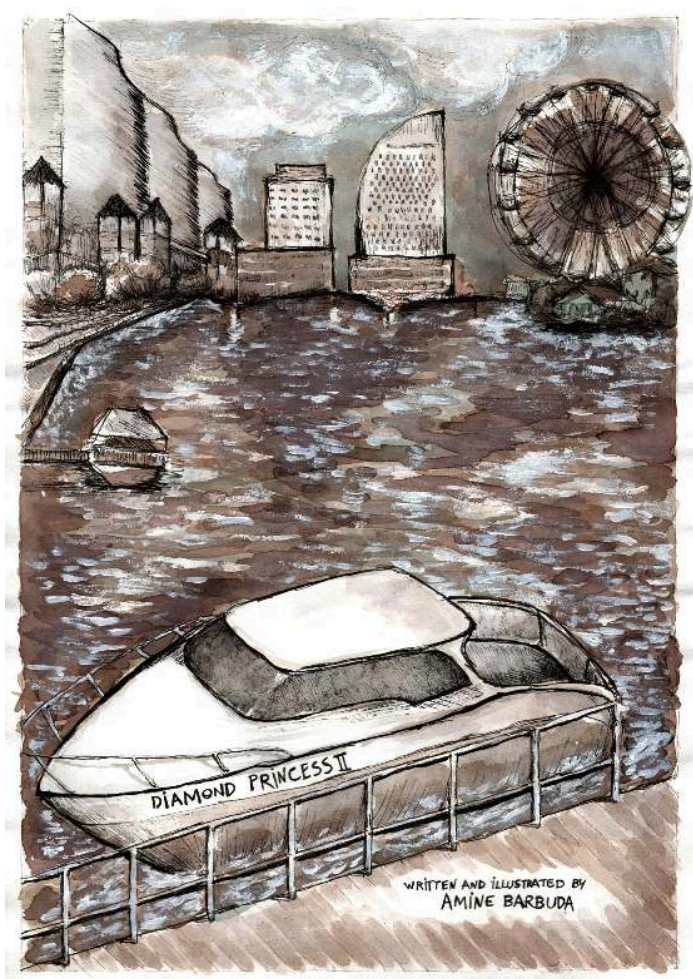

Credits: Amine Barbuda.

Figure 3: Plate 2, Diamond Princess II

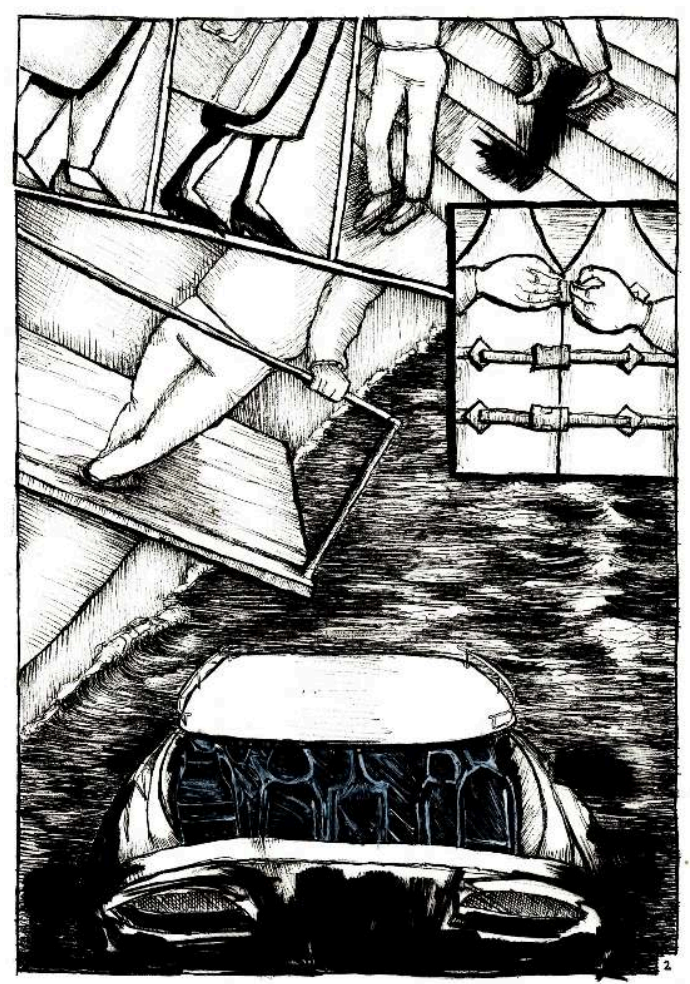

Credits: Amine Barbuda. 
Figure 4: Plate 3, Diamond Princess II

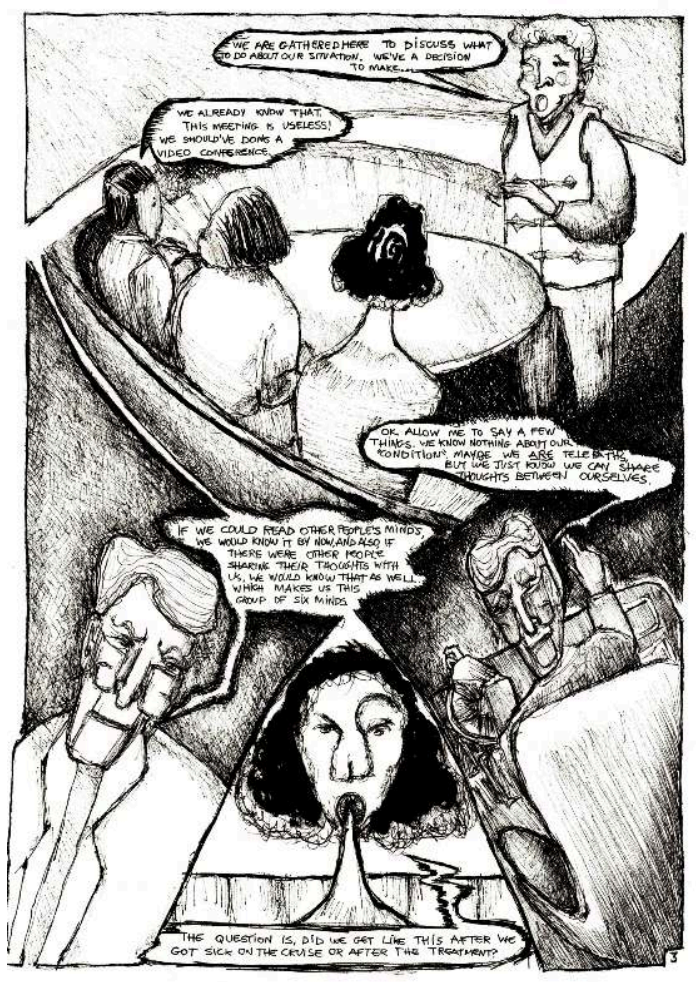

Credits: Amine Barbuda.

Figure 5: Plate 4, Diamond Princess II

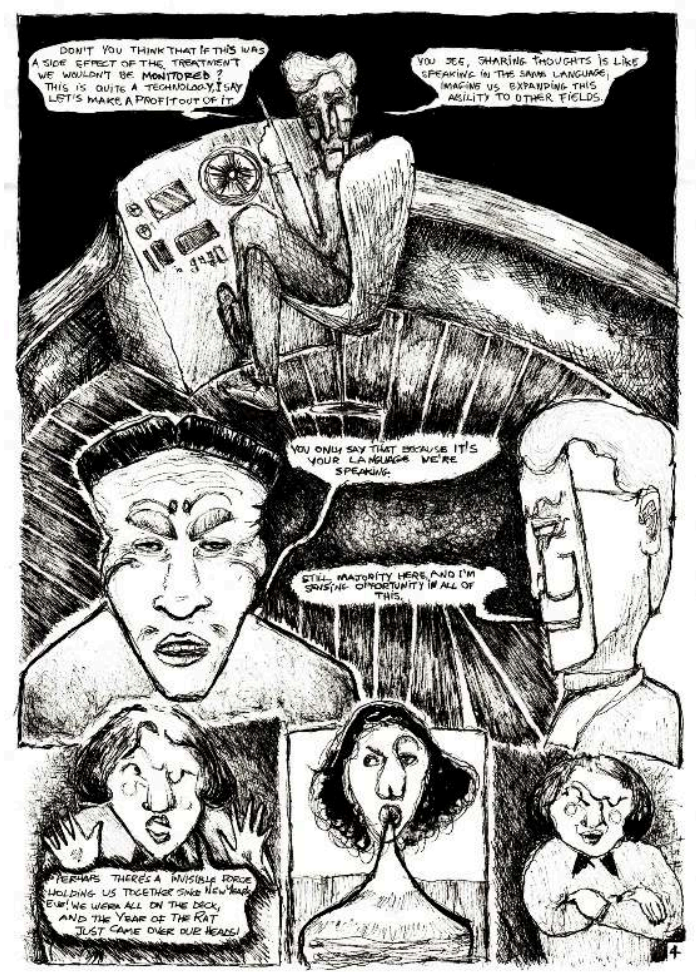

Credits: Amine Barbuda. 
Figure 6: Plate 5, Diamond Princess II

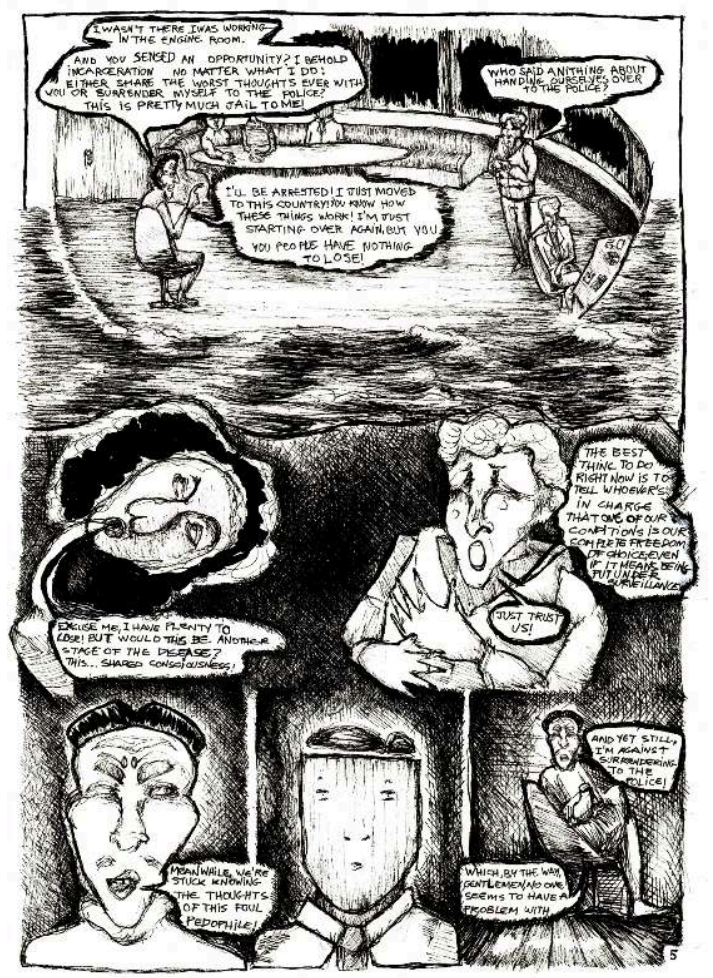

Credits: Amine Barbuda.

Figure 7: Plate 6, Diamond Princess II

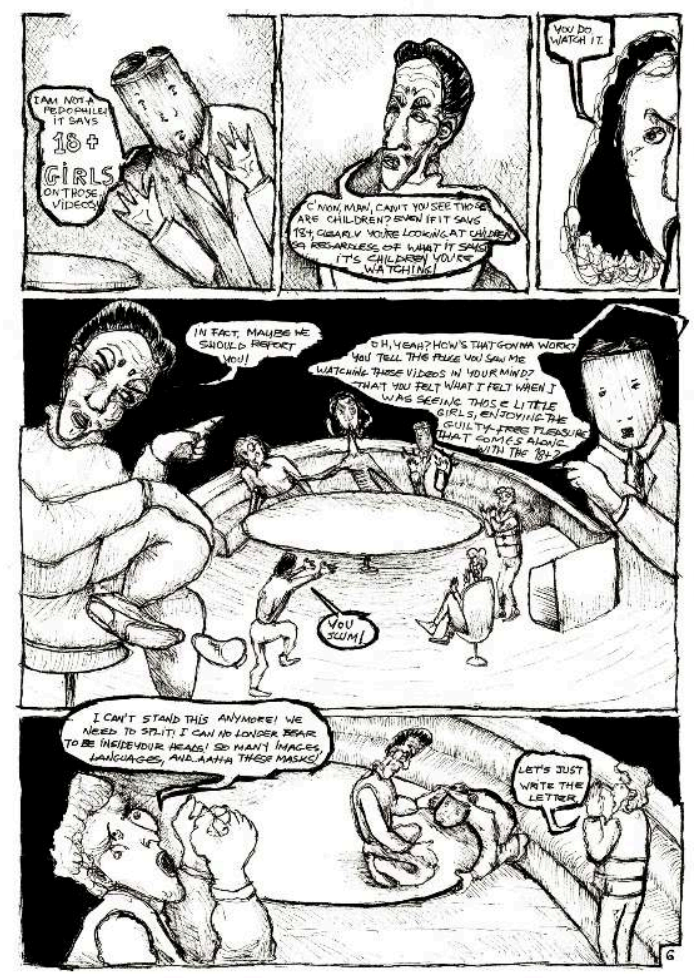

Credits: Amine Barbuda. 
Figure 8: Plate 7, Diamond Princess II

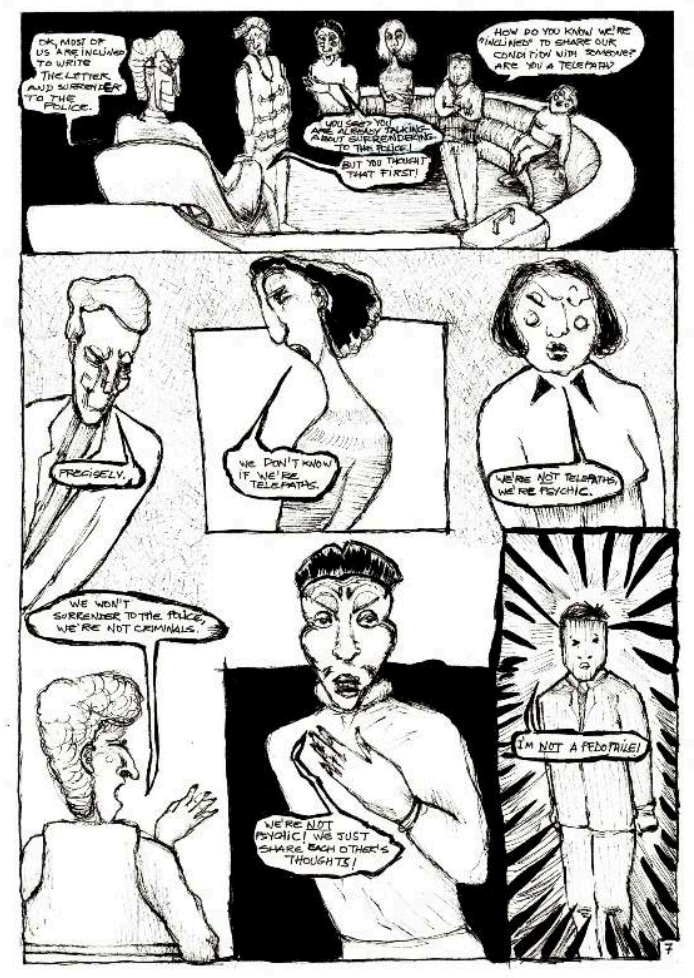

Credits: Amine Barbuda.

Figure 9: Plate 8, Diamond Princess II

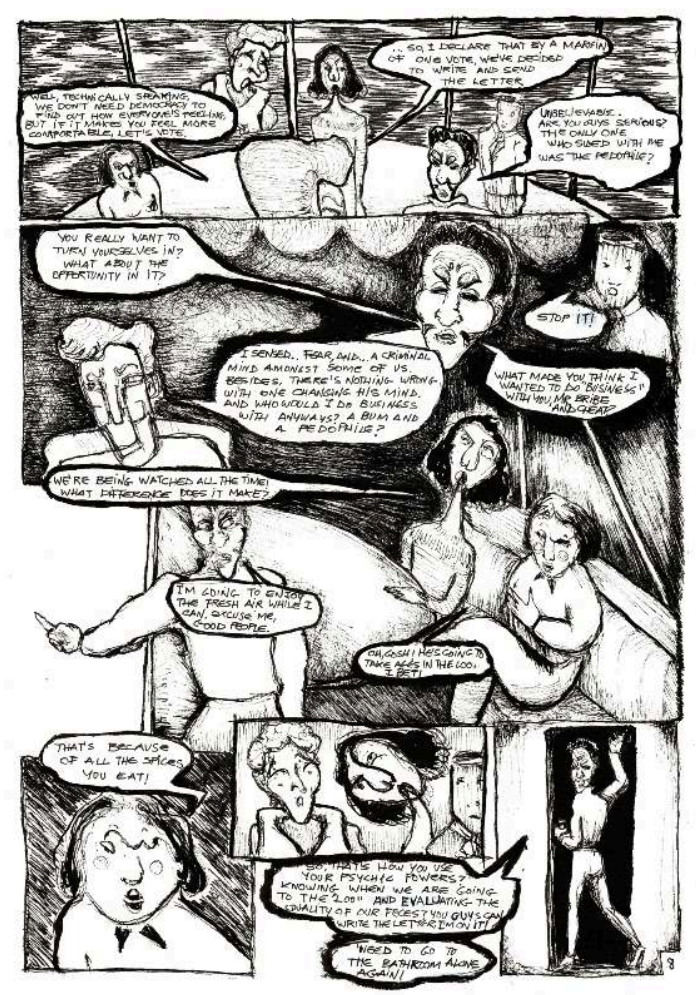

Credits: Amine Barbuda. 
Figure 10: Plate 9, Diamond Princess II

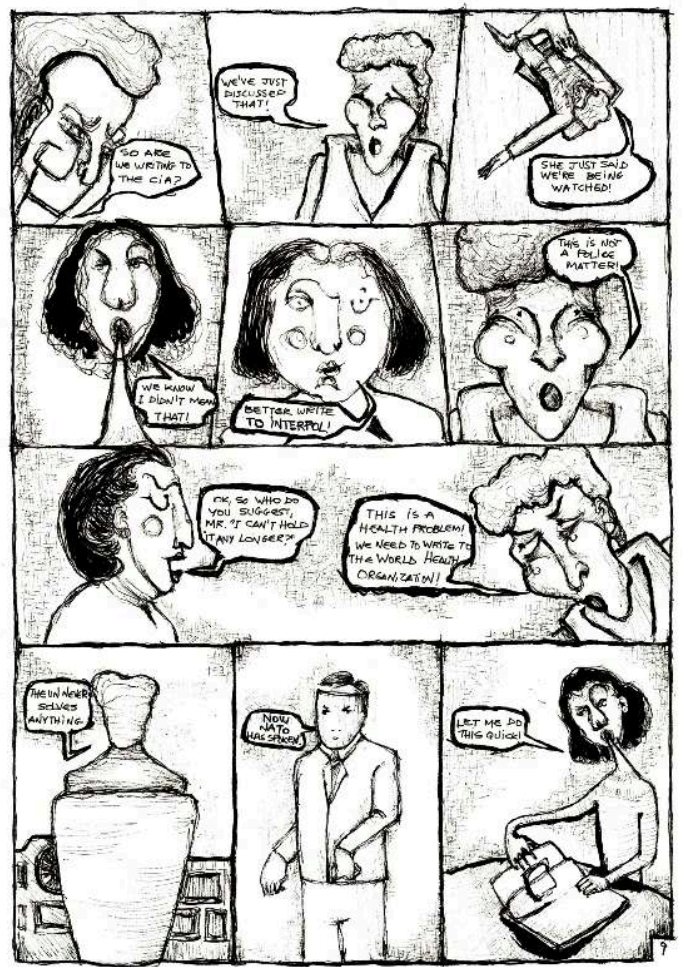

Credits: Amine Barbuda.

Figure 11: Plate 10, Diamond Princess II
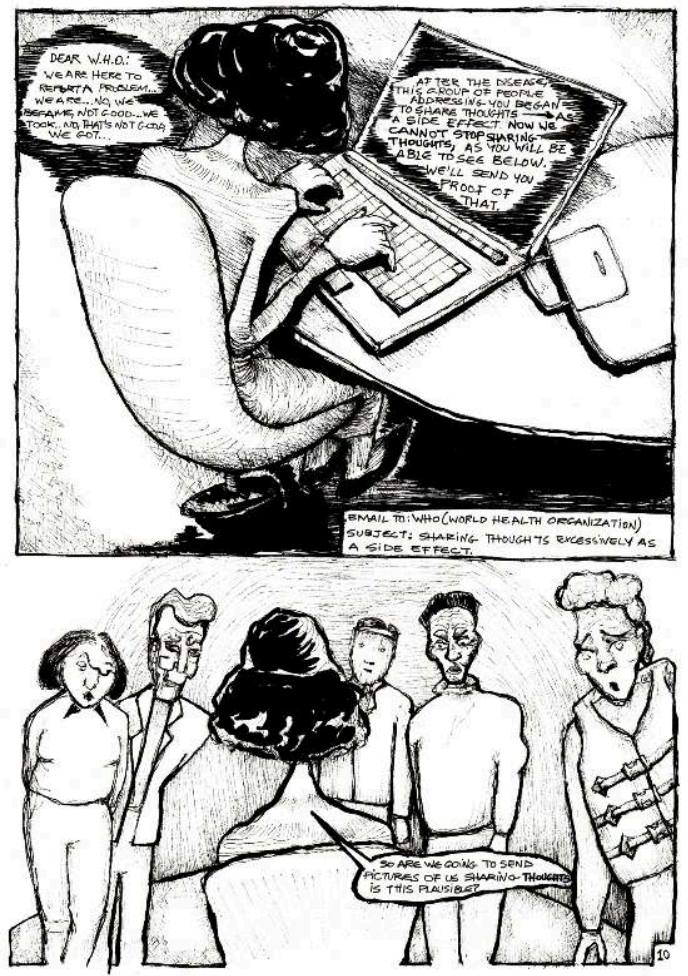

Credits: Amine Barbuda. 
Figure 12: Plate 11, Diamond Princess II

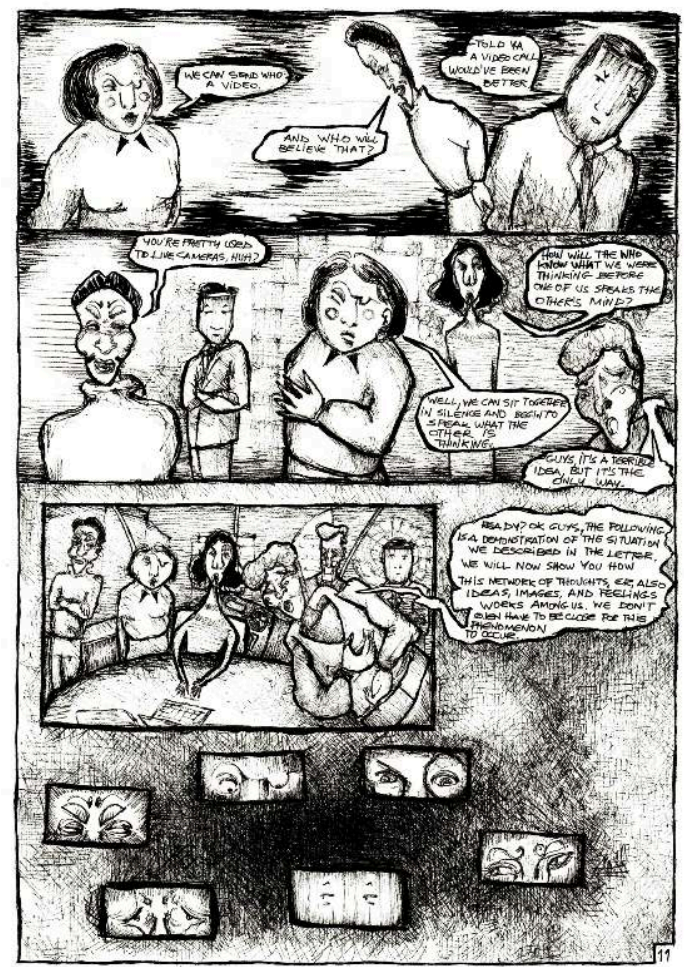

Credits: Amine Barbuda.

Figure 13: Plate 12, Diamond Princess II

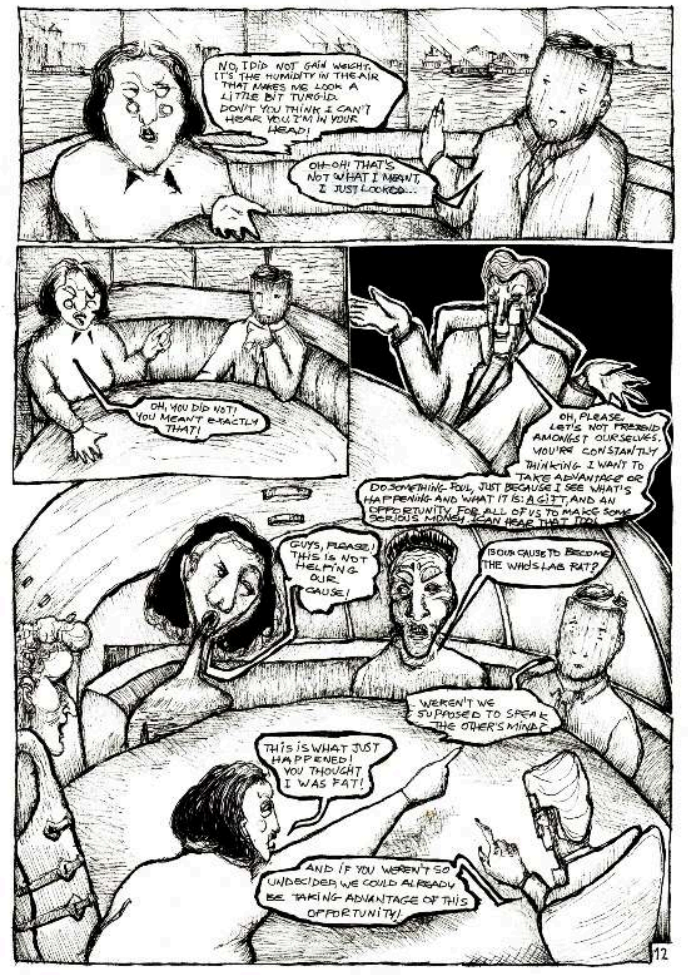

Credits: Amine Barbuda. 
Figure 14: Plate 13, Diamond Princess II

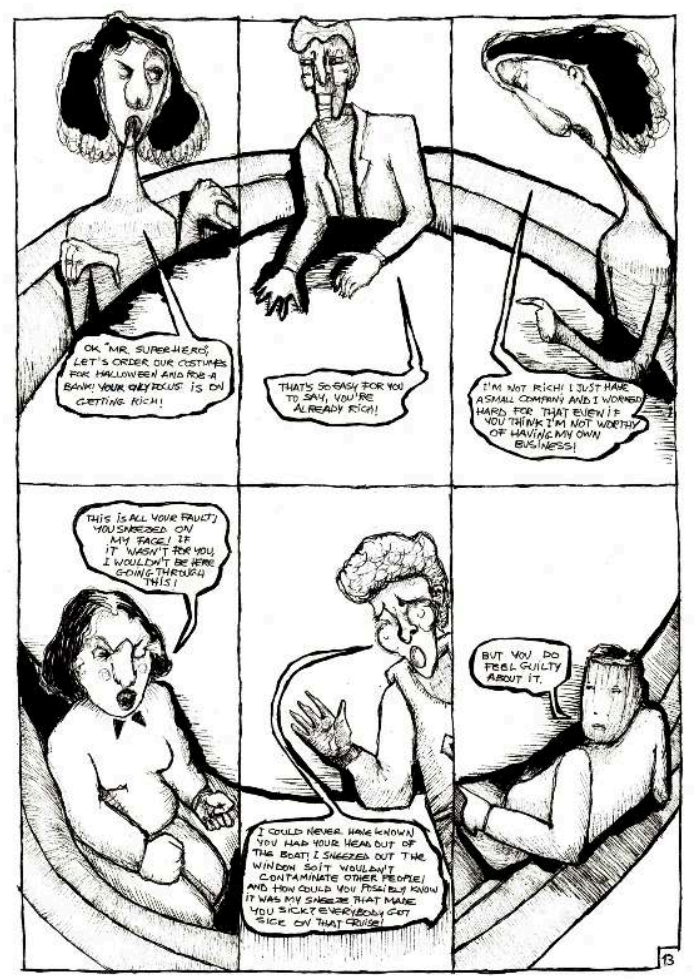

Credits: Amine Barbuda.

Figure 15: Plate 14, Diamond Princess II

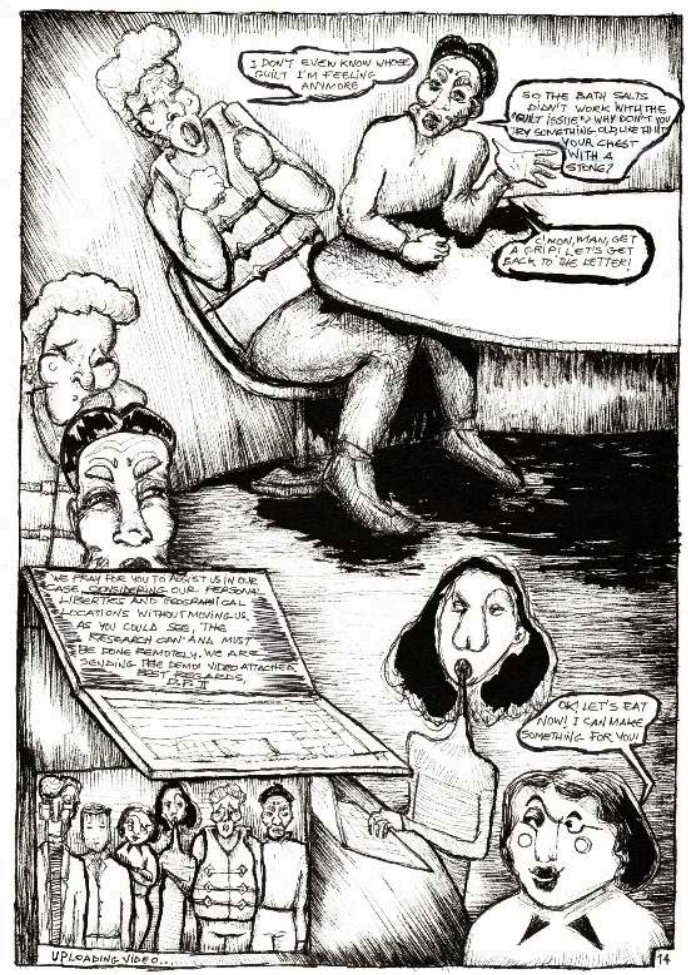

Credits: Amine Barbuda. 
Figure 16: Plate 15, Diamond Princess II
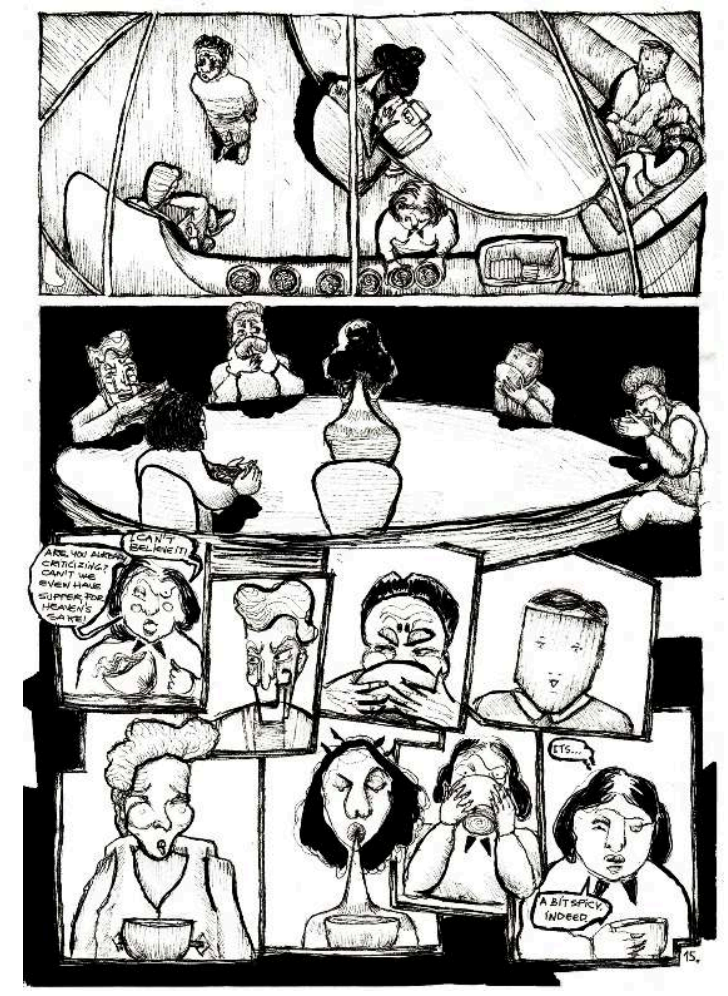

Credits: Amine Barbuda.

Figure 17: Plate 16, Diamond Princess II

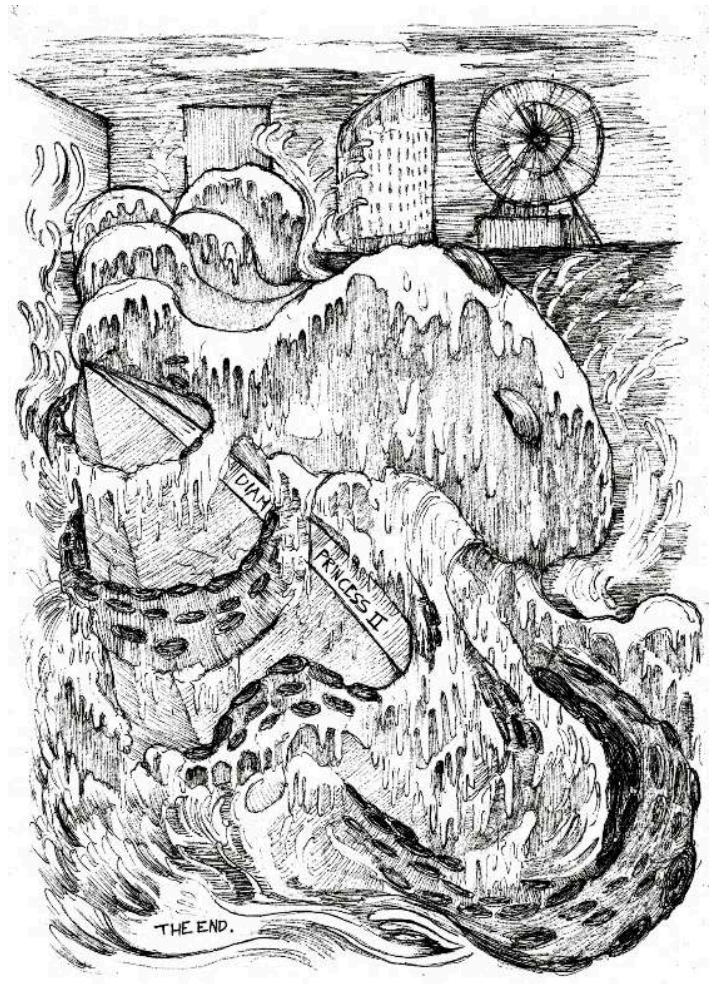

Credits: Amine Barbuda. 
Figure 18: References, Diamond Princess II

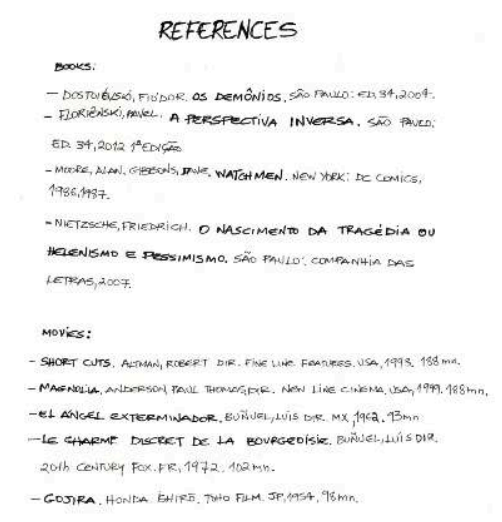

Credits: Amine Barbuda.

BIBLIOGRAPHY

\section{Books}

Dostoiévski, Fiódor. Os Demônios. São Paulo: Ed. 34, 2004.

Floriênski, Pavel. A Perspectiva inversa. São Paulo: Ed. 34, 2012.

Moore, Alan, and Dave Gibbons. Watchmen. New York: DC Comics, 1986-1987.

Nietzsche, Friedrich. O Nascimento da tragédia ou helenismo e pessimismo. São Paulo: Companhia Das Letras, 2007.

\section{Movies}

Short Cuts. Altman, Robert, dir. Fine Line Features. USA, 1993. 188mn.

Magnolia. Anderson, Paul Thomas, dir. New Line Cinema. USA, 1999. 188mn. 
El ángel exterminador. Buñuel, Luís dir. Mexico, 1962. 93mn.

Le charme discret de la bourgeoisie. Buñuel, Luís dir. 20th Fentury Fox. France, 1972. 102mn.

Gojira. Honda Ishiro. Toho Films. Japan, 1954. 98mn.

\section{ABSTRACTS}

This graphic tale takes place among a group of six passengers from the Diamond Princess - a cruise ship which formed a COVID-19 cluster in the beginning of 2020 - who meet later in the city of Yokohama, Japan. They need to discuss a collective condition: a mental communication between them, which they don't know whether it can be attributed to the treatment they received or to the original illness. The main references for this graphic tale are the movies $E l$ ángel exterminador (1962) and Le charme discret de la bourgeoisie (1972), both written and directed by Luís Buñuel. The movies, immersed in a surrealist atmosphere created by the relationships between the characters, bring elements to the script that were observed at the Diamond Princess, such as people held up in a location by an "invisible force," a sense of broken communication, and the spread of misleading information. These are some issues that we now have to face during the pandemic: a combined breakdown and continuous flow of (mis)communication which challenges the referential framework of the world we inhabit, outsourcing reflexive power onto international bodies (such as the WHO), social media (through biographical text and video), or the reader.

Ce récit graphique se déroule au sein d'un groupe de six passagers du Diamond Princess, un bateau de croisière qui connut un cas de contamination collective par COVID-19 au début de l'année 2020, qui se retrouvent plus tard dans la ville de Yokohama, au Japon. Ils doivent discuter d'une pathologie à caractère collectif : une communication mentale entre eux, dont ils ne savent pas si elle est imputable au traitement qu'ils ont reçu ou à la maladie originelle. Les principales références de ce récit graphique sont les films El ángel exterminador (1962) et Le charme discret de la bourgeoisie (1972), tous deux écrits et réalisés par Luís Buñuel. Ces films, plongés dans une atmosphère surréaliste à travers la relation des personnages, apportent au scénario des éléments qui ont été observés au Diamond Princess, tels que des personnes bloquées dans un lieu par une "force invisible», le sentiment d'une communication rompue, et des informations trompeuses. Ce sont là certaines des questions auxquelles nous devons faire face aujourd'hui pendant la pandémie : une rupture parallèle et un flux continu de (mauvaises ou fausses) communications qui remettent en question le cadre référentiel du monde dans lequel nous vivons, tout en externalisant le processus de réflexion vers des organismes internationaux (comme l'OMS) ou les réseaux sociaux (par le biais de textes et de vidéos biographiques), ou encore le lecteur.

\section{INDEX}

Mots-clés: COVID-19, roman graphique, surréalisme, communication, illustration, art, contamination

Keywords: COVID-19, graphic novel, surrealism, communication, illustration, art, contamination 


\section{AUTHOR}

\section{AMINE BARBUDA}

Amine Barbuda is a Brazilian painter, designer, scenographer, architect and urban planner. A graduate of the Faculty of Architecture and Urbanism at the Federal University of Bahia, Barbuda also holds a Master's degree in contemporary urban processes from the same institution. She studies graphic narratives in several languages, graphic novels in different techniques, and painting, especially oil painting. Recently, the artist launched two studies involving dance, performance, composition and painting. See:

http://lattes.cnpq.br/4019471909117612

https://amine.com.br/

https://linktr.ee/AmineBarbuda

https://www.instagram.com/aminebarbuda/

https://p55.com.br/produto/metodos-frankenstein-para-a-criacao-de-uma-barbie/ 